\title{
Observation of microlensing toward the galactic spiral arms. EROS II 3 year survey ${ }^{\star}$
}

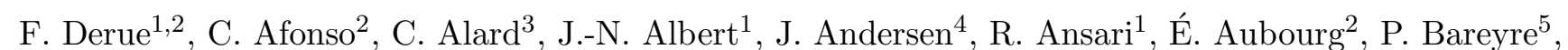

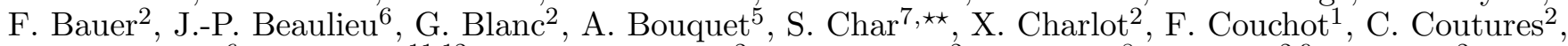
R. Ferlet ${ }^{6}$, P. Fouqué11,12, J.-F. Glicenstein ${ }^{2}$, B. Goldman ${ }^{2}$, A. Gould ${ }^{8}$, D. Graff ${ }^{2,9}$, M. Gros ${ }^{2}$, J. Haïssinski ${ }^{1}$, J.-C. Hamilton ${ }^{5}$, D. Hardin ${ }^{2}$, J. de Kat ${ }^{2}$, A. Kim ${ }^{5}$, T. Lasserre ${ }^{2}$, L. Le Guillou ${ }^{2}$, É. Lesquoy ${ }^{2}$, C. Loup ${ }^{6}$, C. Magneville ${ }^{2}$, B. Mansoux ${ }^{1}$, J.-B. Marquette ${ }^{6}$, É. Maurice ${ }^{10}$, A. Milsztajn ${ }^{2}$, M. Moniez ${ }^{1}$, N. Palanque-Delabrouille ${ }^{2}$, O. Perdereau ${ }^{1}$, L. Prévot ${ }^{10}$, N. Regnault ${ }^{1}$, J. Rich ${ }^{2}$, M. Spiro ${ }^{2}$, A. Vidal-Madjar ${ }^{6}$, L. Vigroux ${ }^{2}$, and S. Zylberajch ${ }^{2}$

1 Laboratoire de l'Accélérateur Linéaire, IN2P3-CNRS, Université de Paris-Sud, BP 34, 91898 Orsay Cedex, France

2 CEA, DSM, DAPNIA, Centre d'Études de Saclay, 91191 Gif-sur-Yvette Cedex, France

3 DASGAL, INSU-CNRS, 77 avenue de l'Observatoire, 75014 Paris, France

4 Astronomical Observatory, Copenhagen University, Juliane Maries Vej 30, 2100 Copenhagen, Denmark

${ }^{5}$ Collège de France, LPCC, IN2P3-CNRS, 11 place Marcellin Berthelot, 75231 Paris Cedex, France

6 Institut d'Astrophysique de Paris, INSU-CNRS, 98bis Boulevard Arago, 75014 Paris, France

7 Universidad de la Serena, Facultad de Ciencias, Departamento de Fisica, Casilla 554, La Serena, Chile

8 Department of Astronomy, Ohio State University, Columbus, Ohio 43210, USA

9 Department of Physics, Ohio State University, Columbus, Ohio 43210, USA

10 Observatoire de Marseille, INSU-CNRS, 2 place Le Verrier, 13248 Marseille Cedex 04, France

11 Observatoire de Paris, DESPA, 92195 Meudon Cedex, France

12 European Southern Observatory (ESO), Casilla 19001, Santiago 19, Chile

Received 4 January 2000 / Accepted 19 March 2001

\begin{abstract}
We present an analysis of the light curves of 9.1 million stars observed during three seasons by EROS (Expérience de Recherche d'Objets Sombres), in the Galactic plane away from the bulge. Seven stars exhibit luminosity variations compatible with gravitational microlensing effects due to unseen objects. The corresponding optical depth, averaged over four directions, is $\bar{\tau}=0.43 \pm 0.2 \times 10^{-6}$. While this value is compatible with expectations from simple Galactic models under reasonable assumptions on the target star distances, we find an excess of events with short timescales toward the direction closest to the Galactic centre. We discuss a possible interpretation involving the contribution of an elongated bar.
\end{abstract}

Key words. galaxy: bar - galaxy: kinematics and dynamics - galaxy: stellar content - galaxy: structure gravitational lensing

\section{Introduction}

Extensive photometric surveys, triggered by Paczyński's suggestion (1986), have led to the observation of microlensing effects toward the Magellanic clouds (EROS, Aubourg et al. 1993; MACHO, Alcock et al. 1993) and

Send offprint requests to: $\mathrm{R}$. Ansari,

e-mail: ansari@lal.in2p3.fr

See also our web server at http://eros.in2p3.fr

* This work is based on observations made with the MARLY telescope at the European Southern Observatory, La Silla, Chile.

$\star \star$ Deceased. the Galactic bulge (OGLE, Udalski et al. 1994; Udalski et al. 2000; MACHO, Alcock et al. 1995; Alcock et al. 1997; Alcock et al. 2000) ${ }^{1}$. The few hundred events observed toward the Galactic centre have strengthened the hypothesis of a barred structure. The early suggestion of de Vaucouleurs (1964) that the Galaxy is barred is now supported by many other observations including photometric measurements (Dwek et al. 1995; Hammersley et al. 2000), studies of gas (Weiner \& Sellwood 1999), stellar

\footnotetext{
1 See also MACHO and OGLE web servers: http://wwwmacho.mcmaster.ca http://www . astrouw.edu.pl/õgle/
} 
kinematics (Zhao et al. 1996) and star counts (Stanek et al. 1994). Nevertheless, the bar parameters (shape, size, mass ...) are not yet precisely known.

In order to improve our knowledge of the Galactic structure, EROS started a dedicated observing programme in four directions of the Galactic plane in 1996. These directions, at large angles from the Galactic centre, have been chosen to disentangle the disc, bar and halo contributions to the optical depth. Three events with long Einstein radius crossing times have already been published, based on two year (1996-97) EROS observations (Derue et al. 1999, hereafter Paper I). Because of their long duration, they are more easily interpreted as lensing events due to disc objects, rather than to halo deflectors. We present in this paper an analysis of the three-year data set (1996-1998).

\section{Experimental setup and observations}

The telescope, camera and observations, as well as the operations and data reduction are described in Paper I and references therein. Four different directions are being monitored in the Galactic plane, corresponding to a total of 29 one-square-degree fields with high stellar densities, covering a wide range of Galactic longitude. The observation parameters for these fields (EROS Galactic Spiral Arms - GSA) are summarized in Table 1. The three year data set contains 9.1 million light curves: 2.1 toward $\beta$ Sct, 1.8 toward $\gamma$ Sct, 3.0 toward $\gamma$ Nor and 2.2 toward $\theta$ Mus. The observations span the period between July 1996 and November 1998, except for $\theta$ Mus which have been monitored only since January 1997. An average of 125 measurements per field were obtained in each of the $R_{\text {EROS }}$ and $V_{\text {EROS }}$ bands, which are related to the Cousins $I$ and Johnson $V$ magnitudes through the following colour equations:

$$
\begin{aligned}
& I_{\mathrm{C}}=R_{\mathrm{EROS}}-0.80+0.01 \times\left(V_{\mathrm{EROS}}-R_{\mathrm{EROS}}\right) \\
& V_{\mathrm{J}}=V_{\text {EROS }}-0.37+0.39 \times\left(V_{\mathrm{EROS}}-R_{\mathrm{EROS}}\right) .
\end{aligned}
$$

The colour coefficients were obtained from the study of our passbands, and the zero points were established with data taken at the ESO-Danish telescope (Regnault 2000). We cross-checked that, with these colour equations, the mean magnitudes of the LMC red-giant clump stars agree within 0.1 magnitude precision, with determinations by Harris \& Zaritsky (1999) and Udalski et al. (1998).

Figure 1 shows the field positions in Galactic coordinates, while Fig. 2 represents the observation time span and average sampling for the different directions.

\section{The search for lensed stars}

\subsection{Data analysis and event selection}

The data analysis is similar to that of the first two years, except that no criteria based on the colour-magnitude diagram were needed. This change was made possible by the
Table 1. Characteristics of the 29 fields monitored in the EROS spiral arms program. Positions of the field centres, average sampling (number of photometric measurements per light curve per colour) and number of analysed light curves are indicated for each field. Field gn401 has not been studied yet.

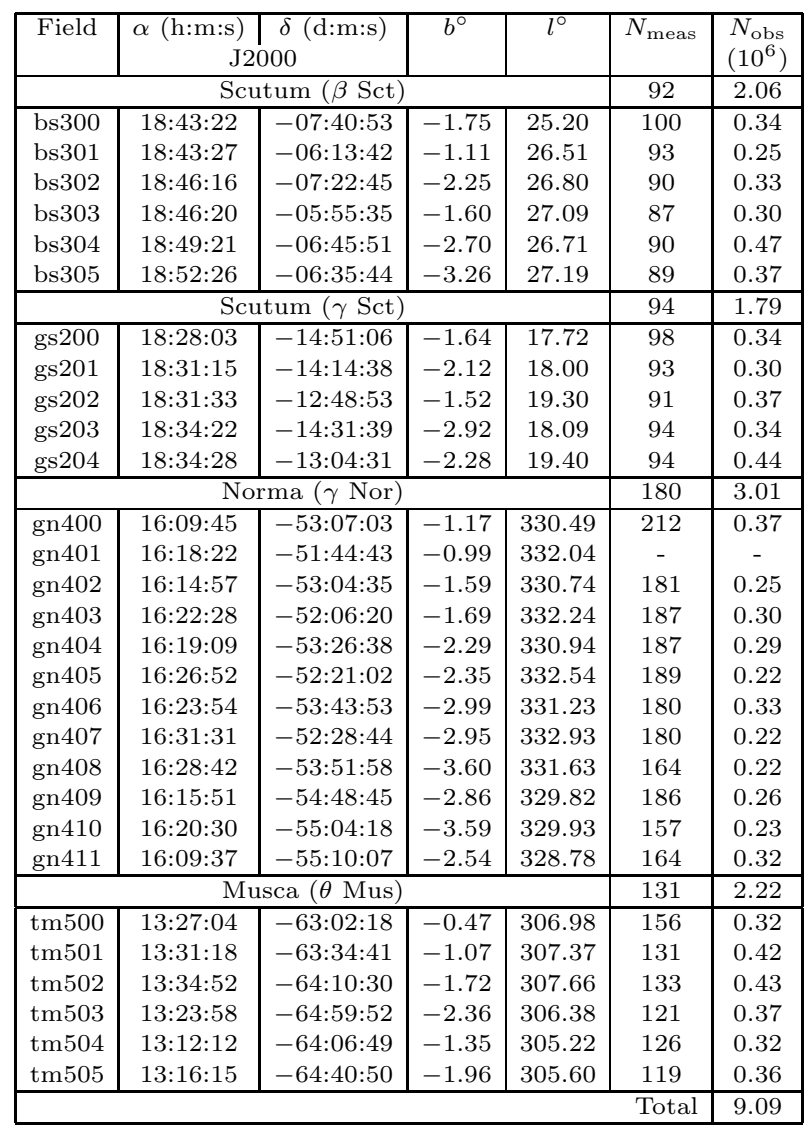

longer time coverage, allowing a better rejection against variable stars.

Light curves for both colours have been produced from the sequences of images using the specific software PEIDA (Photométrie et Étude d'Images Destinées à l'Astrophysique), designed to extract photometric information in crowded fields (Ansari 1996). The efficiency of the selection process described below has been controlled with Monte-Carlo generated light curves (see Sect. 3.2). The resulting efficiencies and rejection factors are summarized in Table 2.

- After producing the light curves for a total of 9085921 sources, the first step of the event selection filter consists in a non specific prefiltering that retains the $\sim 13 \%$ most variable light curves, satisfying at least one of the following criteria: the strongest fluctuation along the light curve (series of consecutive flux measurements that lie below or above the base line) is incompatible with a stable light curve; or the dispersion of the flux measurements is significantly larger than expected from their estimated photometric precision; or the distribution of the deviations with respect to the base flux is incompatible with the one expected from the 


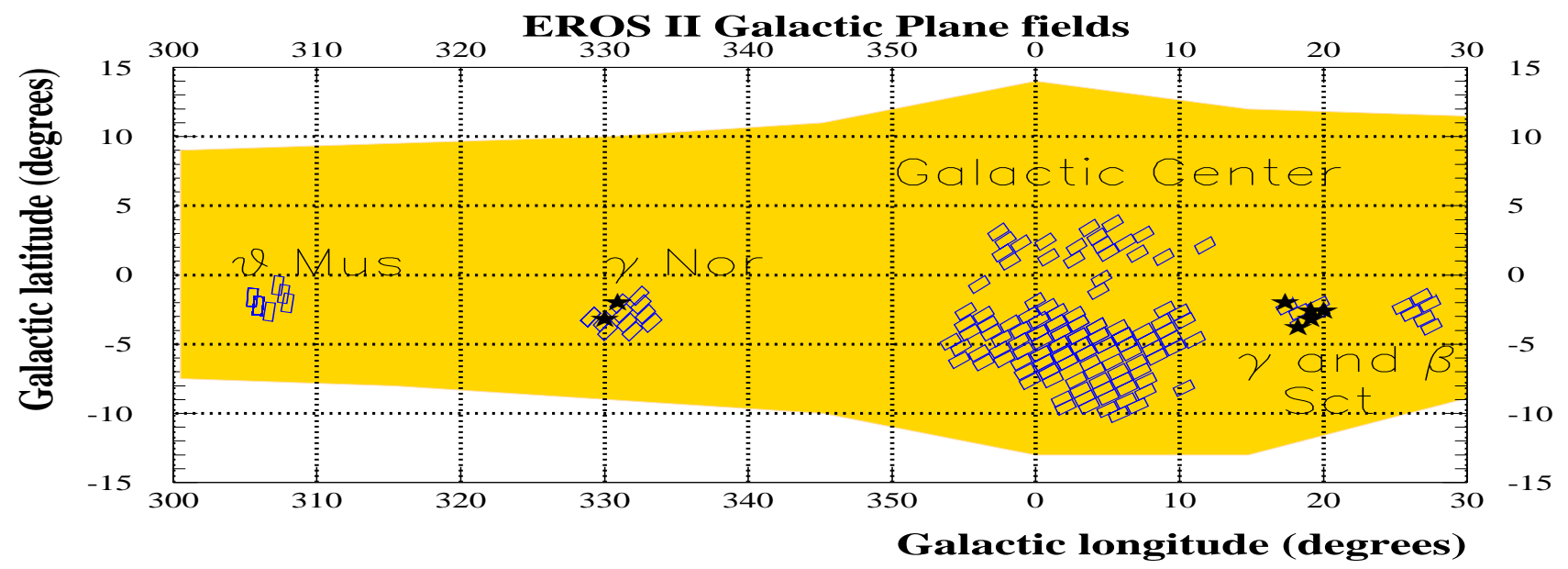

Fig. 1. Map of the Galactic plane fields (Galactic coordinates) monitored by EROS for the microlensing search. The shaded area represents the shape of the Galaxy. The positions of our fields toward the spiral arms, as well as our Galactic bulge fields (not discussed in this paper) are shown. The stars $(\star)$ indicate the positions of our seven microlensing candidates.

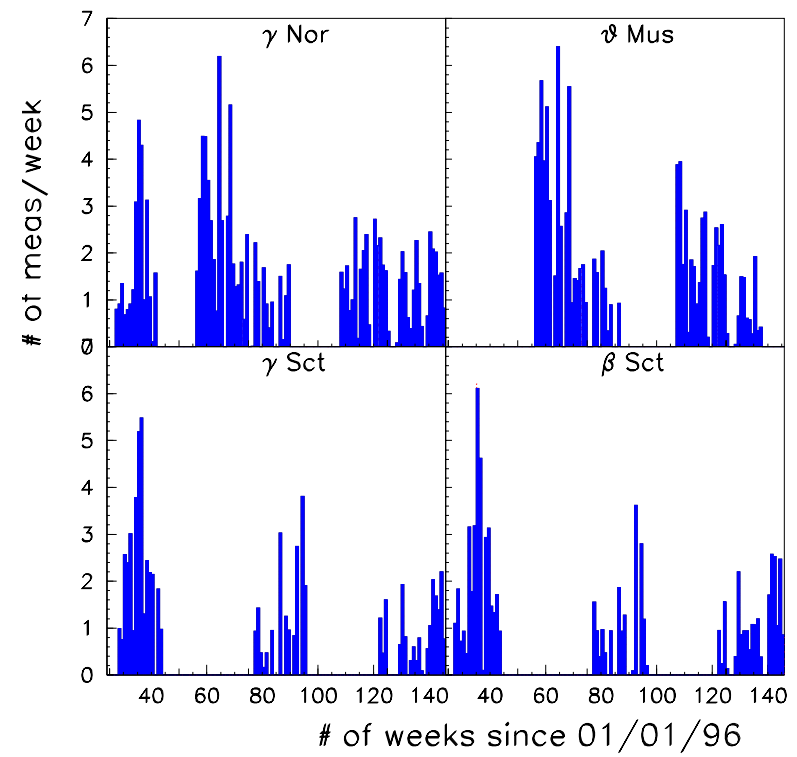

Fig. 2. Time sampling for each direction monitored toward the spiral arms, in number of measurements per week since 1 Jan. 1996.

measurements of a stable source with Gaussian errors from a Kolmogorov-Smirnov test. The thresholds of these three criteria have been tuned to select a total of $\sim 13 \%$ of the light curves. We also include a randomly selected set of light curves $(\sim 2 \%)$ to produce unbiased colour-magnitude diagrams and for use in simulations;

- We then search for bumps in each light curve. A bump is defined as a series of consecutive flux measurements that starts with a positive fluctuation of more than one standard deviation $(+1 \sigma)$ from the base flux, ends when 3 consecutive measurements lie below $+1 \sigma$ from the base line and contains at least four measurements deviating by more than $+1 \sigma$. A probability $Q$ is associated to each bump assuming gaussian errors and a stable source. We require that the light curve contains at least one such bump for each colour. Then we
Table 2. Effect of the selection criteria on data and MonteCarlo. For each cut, the number of remaining light curves (data) and the efficiency (Monte-Carlo) is given. The efficiencies correspond to the fraction of remaining simulated events accepted by each selection criterion, averaged over all four targets and over the simulation parameters.

\begin{tabular}{lrc}
\hline Criterion & Data & Monte-Carlo \\
\hline Total analysed & 9085921 & - \\
pre-filtering & 1413408 & \} $39 \%$ \\
significant bumps in $R \& B$ & 208125 & \\
bumps $R$ and $B$ overlap & 105130 & $85 \%$ \\
second bump small & 26470 & $79 \%$ \\
$\frac{\rho \sqrt{N-1}}{\sqrt{1-\rho^{2}}}<9$. & 24924 & $97 \%$ \\
$\chi_{\mathrm{ml}-\text { out }}^{2}<8$. & 17270 & $84 \%$ \\
$\Delta \chi^{2}>15$. & 20 & $54 \%$ \\
$u_{0}<1$. & 11 & $88 \%$ \\
$t_{\mathrm{E}}>1$ day & 7 & $100 \%$ \\
\hline
\end{tabular}

require a minimum overlap between the most significant bumps (main bumps) in each colour. The ratio of the overlap time to the joined time of the two bumps should exceed $10 \%$;

- The next two criteria are intended to remove repetitive variable stars. For this purpose we require the probability $Q$ of the second most significant bump (if any) to be significantly larger than that of the first bump (more than a factor $\sim 3$ ). We also demand the correlation between the red and blue light curves outside the main bump to be small: the correlation coefficient $\rho$ is estimated from blue and red measurements that do not belong to the main fluctuation. We require that $\frac{\rho \sqrt{N-1}}{\sqrt{1-\rho^{2}}}<9$., where $N$ is the number of pairs of simultaneous measurements. At this stage, only light curves with no significant fluctuation or with uncorrelated fluctuations outside the main bump remain. We then perform a microlensing fit on the selected light curves; 


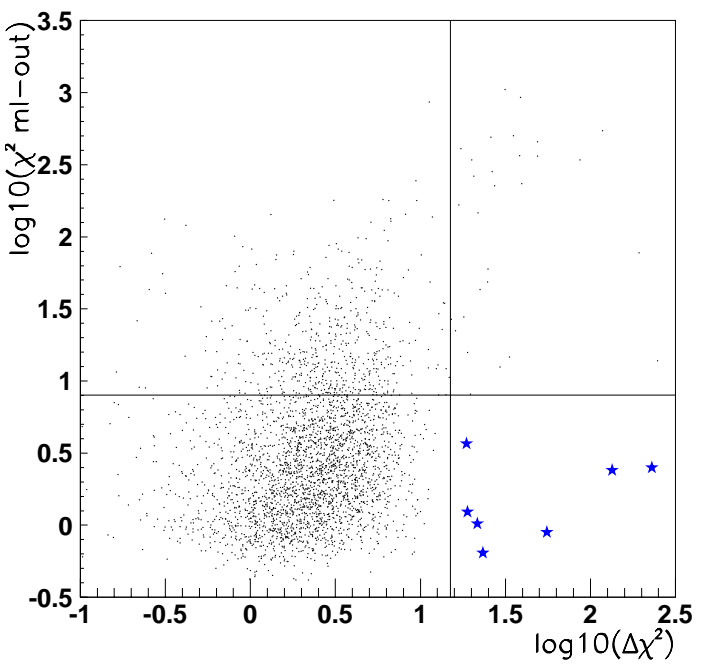

Fig. 3. Distribution of $\log _{10}\left(\chi_{\mathrm{ml}-\text { out }}^{2}\right)$ versus $\log _{10}\left(\Delta \chi^{2}\right)$ for the light curves that satisfy all other selection criteria. The two lines correspond to the adopted cuts. Stars $(\star)$ correspond to the seven candidates selected by our analysis.

- The next two variables are introduced to quantify the quality of the microlensing fit: we estimate $\chi_{\mathrm{ml}-\mathrm{out}}^{2}$ as the combined $\chi^{2}$ per degree of freedom of the microlensing fit for both colours, estimated outside the hypothetical microlensing peak (i.e. restricted to periods where the fitted magnification is lower than 10\%). We reject light curves with $\chi_{\text {ml-out }}^{2}>8$. We retain only high signal-to-noise ratio events by requiring a significant improvement of the microlensing fit $(\mathrm{ml})$ over a constant flux fit (cst), in both colours:

$$
\begin{aligned}
\Delta \chi_{B, R}^{2} & =\left.\frac{\chi_{\mathrm{cst}}^{2}-\chi_{\mathrm{ml}}^{2}}{\chi_{\mathrm{ml}}^{2} / N_{\mathrm{dof}}} \frac{1}{\sqrt{2 N_{\mathrm{dof}}}}\right|_{B, R} \\
\Delta \chi^{2} & =\operatorname{Min}\left(\Delta \chi_{B}^{2}, \Delta \chi_{R}^{2}\right)>15 ;
\end{aligned}
$$

- In the last step of our selection process, we retain light curves with a fitted impact parameter $u_{0}<1$. and a fitted Eintein radius crossing time $t_{\mathrm{E}}>1$ day.

Seven light curves satisfy all requirements; they are labelled EROS-GSA1 to 7. Figure 3 shows the distribution of $\log _{10}\left(\chi_{\mathrm{ml}-\text { out }}^{2}\right)$ versus $\log _{10}\left(\Delta \chi^{2}\right)$ for light curves satisfying all the other selection criteria. The seven candidates are located in a region of the diagram corresponding to lightcurves with a magnification well described by a microlensing fit and constant outside the peak. The upper right side of the diagram is populated by variable stars, mostly red and bright. The upper panel of Fig. 4 shows the distribution of $\log _{10}\left(\Delta \chi^{2}\right)$ for the light curves that satisfy all the other criteria. The lower panel gives the estimated optical depth as a function of the minimum threshold on this variable. The estimate of the optical depth does not vary significantly with the cut threshold on $\Delta \chi^{2}$ down to the value $\sim 12$. This indicates that a possible background contamination would have a small impact on our optical depth estimates.
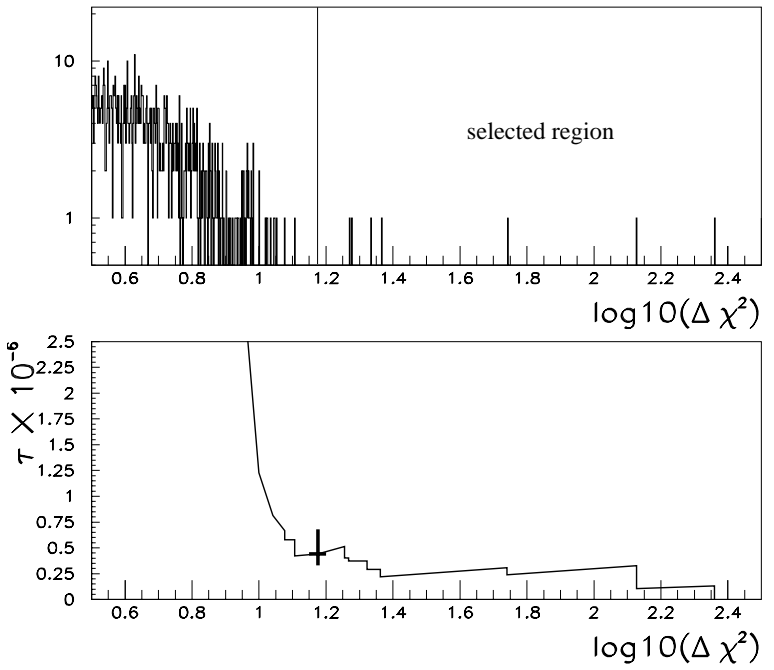

Fig. 4. Upper panel: distribution of $\log _{10}\left(\Delta \chi^{2}\right)$ for the light curves satisfying all the other criteria. Lower panel: estimated average optical depth as a function of the threshold on $\Delta \chi^{2}$. The cross shows the value obtained for the adopted threshold $\left(\Delta \chi^{2}=15\right)$.

\subsection{The selection efficiency}

To determine the efficiency of each selection criterion, we have applied them to Monte-Carlo generated microlensing light curves, obtained from a representative sample of the observed light curves on which we superimpose randomly generated microlensing effects. For this purpose, we use the randomly selected set of light curves from the prefilter stage. The microlensing parameters are uniformly drawn from the following intervals: impact parameter expressed in units of the Einstein radius $u_{0} \in[0,2]$, maximum magnification time in a search period $T_{\text {search }}$ starting 150 days before the first observation and ending 150 days after the last observation and Einstein radius crossing time $t_{\mathrm{E}} \in[1,250]$ days.

For each generated light curve, the base flux is taken to be equal to the randomly selected star's flux. The light curve is also used to reproduce the experiment's time sampling, as well as the photometric errors, taking into account each measurement's observing conditions (seeing, sky background...). Figure 5 shows the variation of the average detection efficiency $\epsilon\left(t_{\mathrm{E}}\right)$ as a function of the event duration $t_{\mathrm{E}}$, for the four monitored directions. As in Paper I, the reported efficiency $\epsilon\left(t_{\mathrm{E}}\right)$ (called sampling efficiency) is relative to a set of unblended stars and normalised to $u_{0}<1$. It is difficult to assess at this stage the impact of blending in our analysis, as the population and distance of the observed stars are poorly known. However, the effect of the blending on our detection efficiency has been found to be less than $20 \%$ toward the Magellanic clouds, due to our relatively low limiting magnitude.

\section{The 7 candidates}

Table 3 contains the characteristics of the 7 candidate microlensing light curves (GSA1-GSA7). Figures 7 and 8 
Table 3. Characteristics of the 7 microlensing candidates: target, microlensing exposure $N_{\text {obs }} T_{\text {search }}$, equatorial and Galactic coordinates, magnitudes, number of measurements, time span, results from the unblended fit; time of the maximum $t_{0}$ (in Julian Day -2447891.5$)$, Einstein radius crossing time $t_{\mathrm{E}}$, maximum magnification $A_{\max }, \chi^{2}$ of the best fit; sampling efficiency $\epsilon$ for events of duration $t_{\mathrm{E}}$ and contribution to each direction's optical depth.

\begin{tabular}{llllllll}
\hline Candidate & GSA1 & GSA2 & GSA3 & GSA4 & GSA5 & GSA6 & GSA7 \\
\hline Field & gs200 & gn400 & gn409 & gs202 & gs202 & gs203 & gs203 \\
$N_{\text {meas }}$ & 263 & 435 & 432 & 190 & 191 & 175 & 188 \\
Time span (days) & 870 & 870 & 870 & 870 & 870 & 870 & 870 \\
$N_{\text {obs }} T_{\text {search }}$ & 5.73 & 9.64 & 9.64 & 5.73 & 5.73 & 5.73 & 5.73 \\
(in 10 ${ }^{6}$ years) & & & & & & & \\
\hline$\alpha($ h:m:s) J2000 & $18: 29: 09.0$ & $16: 11: 50.2$ & $16: 16: 26.7$ & $18: 32: 26.0$ & $18: 32: 12.0$ & $18: 33: 45.5$ & $18: 35: 12.4$ \\
$\delta($ d:m:s) J2000 & $-14: 15: 09$ & $-52: 56: 49$ & $-54: 37: 49$ & $-12: 56: 04$ & $-12: 55: 16$ & $-14: 41: 41$ & $-14: 56: 27$ \\
$l^{\circ}$ & 17.74 & 330.47 & 329.80 & 19.27 & 19.26 & 17.86 & 17.80 \\
$b^{\circ}$ & -1.63 & -1.14 & -2.83 & -1.73 & -1.68 & -2.83 & -3.25 \\
$V_{\mathrm{J}}-I_{\mathrm{C}}$ & $20.7-18.2$ & $20.1-17.7$ & $19.0-17.5$ & $19.3-17.2$ & $20.7-17.9$ & $19.0-17.2$ & $19.0-17.5$ \\
\hline$t_{0}$ & $2408.65 \pm 0.04$ & $2642.9 \pm 0.2$ & $2806.2 \pm 1.1$ & $2406.8 \pm 0.7$ & $2408.4 \pm 2.8$ & $2843.6 \pm 2.9$ & $3201.6 \pm 0.2$ \\
$t_{\mathrm{E}}($ days $)$ & $71.5 \pm 1.1$ & $98.3 \pm 0.9$ & $70.0 \pm 2.0$ & $23.9 \pm 1.1$ & $59.0 \pm 5.1$ & $37.9 \pm 5.0$ & $5.40 \pm 0.30$ \\
$A_{\text {max }}$ & $26.5 \pm 0.6$ & $3.05 \pm 0.02$ & $1.89 \pm 0.01$ & $1.72 \pm 0.02$ & $1.71 \pm 0.03$ & $1.35 \pm 0.02$ & $2.40 \pm 0.30$ \\
$\chi^{2}$ & 233 & 551 & 445 & 357 & 167 & 121 & 195 \\
\hline$\epsilon\left(t_{\mathrm{E}}\right)(\%)$ & 11. & 29. & 25. & 6.7 & 10. & 8.3 & 4. \\
Contribution & & & & & & & \\
to $\tau\left(\times 10^{6}\right)$ & 0.49 & 0.15 & 0.12 & 0.27 & 0.44 & 0.34 & 0.10 \\
\hline
\end{tabular}

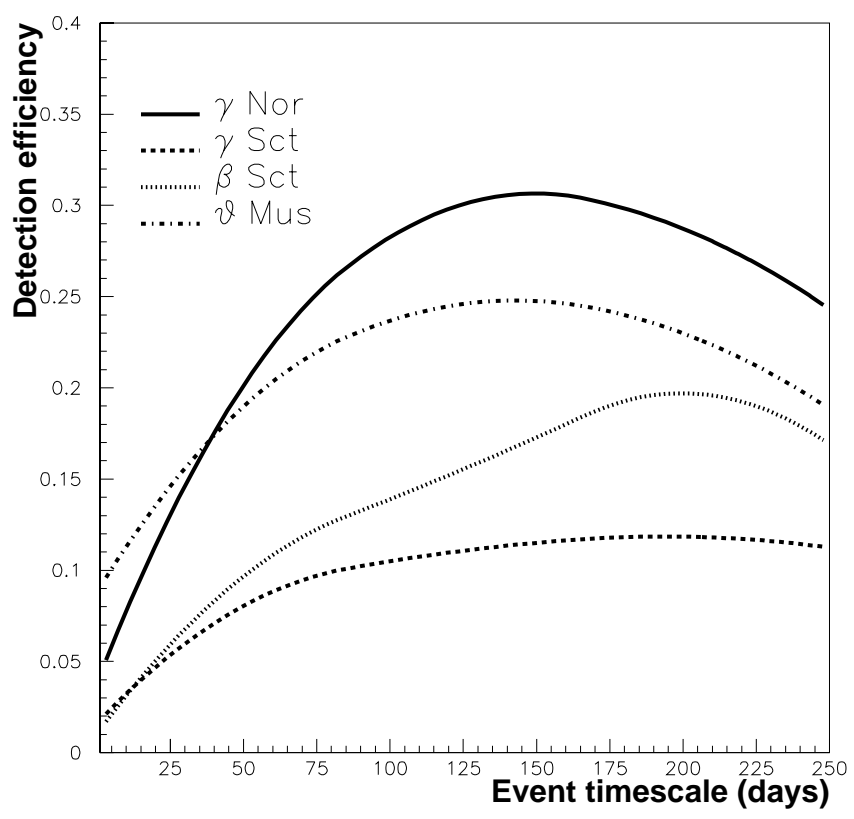

Fig. 5. Average selection efficiency $\epsilon\left(t_{\mathrm{E}}\right)$ as a function of the Einstein radius crossing time $\left(t_{\mathrm{E}}\right)$. The detection efficiency is the ratio of the number of simulated events satisfying the selection criteria - with duration $t_{\mathrm{E}}$, any $u_{0}$ and any date of maximum within the search period - to the number of events generated with $u_{0} \leq 1$.

show their light curves in both EROS passbands. A plot of the correlation between the magnifications in the two colors is also included. The positions of the seven candidates in the colour-magnitude diagrams are shown in Fig. 6. They are representative of the monitored stars, located in the main sequence part of the diagram. Because of the high stellar densities of the fields monitored in microlensing surveys, the flux of each reconstructed star can result from the superposition of the fluxes of many source stars. To take into account this possibility, we performed another microlensing fit with two additional parameters, namely the blending coefficients $\left.c_{\mathrm{bl}}\right|_{B, R}$ in each colour, representing the relative contribution of the lensed star flux to the reconstructed base flux:

$\left.\left.c_{\mathrm{bl}}\right|_{B, R} \equiv \frac{f_{\mathrm{star}}}{f_{\mathrm{star}}+f_{\mathrm{blend}}}\right|_{B, R}$.

The blending coefficient $c_{\mathrm{bl}}$ is unity when there is no blending and $c_{\mathrm{bl}} \rightarrow 0$ in the limit where the magnified star does not contribute at all to the total recovered baseline flux. The fit with blending has not been performed for GSA2, which is compatible with a binary source event (see Paper I). For GSA1, GSA4, GSA5 and GSA7, we find that blending is negligible $\left(\left.c_{\mathrm{bl}}\right|_{B, R}>0.9\right)$. Taking into account a possible blending within the uncertainties has a negligible impact on the contribution of these events to the optical depth $\tau$. For GSA3 and GSA6, the blending coefficients are less constrained, because of the sparse sampling during the phases of fast changing magnification. We find nevertheless that $\left.c_{\mathrm{bl}}\right|_{B, R}>0.3$ for GSA3, corresponding to $t_{\mathrm{E}}<100$ days. The GSA3 contribution to $\tau$ would be increased by $30 \%$ for the maximum allowed blending. The fit to GSA6 light curve shows no blending, but with a very large uncertainty. The values given for the event duration and its contribution to $\tau$ should thus be considered as lower limits.

GSA1 \& 2 have been studied in detail in Paper I leading to additional constraints on lens masses and distances. None of the new candidates shows any noticeable deviation from standard microlensing curves, nor do they 

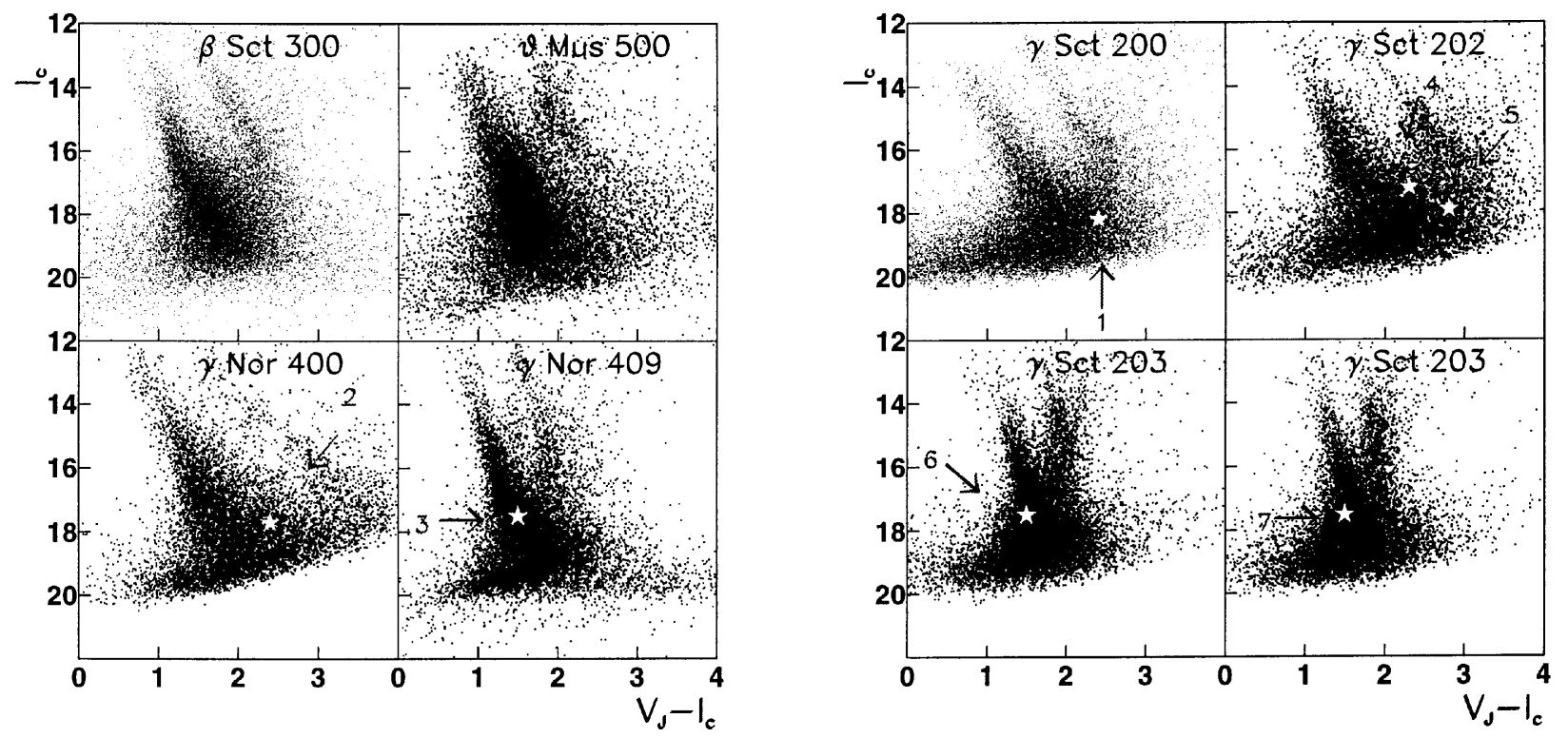

Fig. 6. Colour-magnitude diagrams $\left(I_{\mathrm{C}}\right.$ vs. $\left.V_{\mathrm{J}}-I_{\mathrm{C}}\right)$ for the stars monitored by EROS in the neighbourhood of our candidates. The positions of the 7 candidates are indicated. Typical CM diagrams toward $\beta$ Sct and $\theta$ Mus where no candidate have been found, are also shown.

contain precise enough data to establish useful limits from this lack of deviation.

\section{Optical depth estimation}

For a given target, an estimate of the optical depth - the probability of observing a magnification larger than 1.34 for a pointlike source - can be computed using the expression:

$\tau=\frac{1}{N_{\text {obs }} T_{\text {search }}} \frac{\pi}{2} \sum_{\text {events }} \frac{t_{\mathrm{E}}}{\epsilon\left(t_{\mathrm{E}}\right)}$.

This expression can also be written in the form:

$\tau=\frac{1}{N_{\text {obs }} T_{\text {search }}} \frac{\pi}{2} N_{\text {evt }}\left\langle\frac{t_{\mathrm{E}}}{\epsilon}\right\rangle$

where $N_{\text {obs }}$ is the number of monitored stars in the target and $T_{\text {search }}$ is the duration of the search period $\left(T_{\text {search }}=\right.$ time $\operatorname{span}+300$ days $)$. For this 3 year analysis, $T_{\text {search }}$ is 1170 days toward $\gamma$ Nor, $\gamma$ Sct, $\beta$ Sct and 990 days toward $\theta$ Mus. $\left\langle t_{\mathrm{E}} / \epsilon\right\rangle=\left\langle t_{\mathrm{E}} / \epsilon\left(t_{\mathrm{E}}\right)\right\rangle$ denotes the mean value of event durations weighted by the inverse of the efficiency. As already noted by different authors (see e.g. Han \& Gould 1995) two factors contribute to the uncertainty of the optical depth computed using the above formulae: Poisson fluctuations in the total number of observed events $\sigma\left(N_{\text {evt }}\right)=\sqrt{N_{\text {evt }}}$ and fluctuations in the observed values of $\left\langle t_{\mathrm{E}} / \epsilon\right\rangle$. Given the small statistics, the increase of the error bars due to the latter contribution was found negligible.

The contribution of each event to the optical depth is given in Table 3 . We obtain $\tau(\gamma$ Nor $)=0.27 \times 10^{-6}$ and $\tau(\gamma$ Sct $)=1.64 \times 10^{-6}$ for the two directions where the 7 events have been observed. These values are shown in
Fig. 9, with error bars which include only Poisson fluctuations, corresponding to the classical $68 \%$ confidence intervals (Feldman \& Cousins 1998).

We have also computed an average optical depth $\bar{\tau}$ by a weighted mean $\left(w=N_{\text {obs }} \times T_{\text {search }}\right)$ over the four directions. We find $\bar{\tau}=0.43 \pm 0.2 \times 10^{-6}$ in agreement with expectations from the models described below.

For the $\beta$ Sct and $\theta$ Mus directions, where no event has been observed $\left(N_{\text {evt }}=0\right)$, we compute an upper limit on the optical depth using the expected value of $\left\langle t_{\mathrm{E}} / \epsilon\right\rangle$. This value is computed from the predicted $t_{\mathrm{E}}$ distribution for model 1 (see Sect. 6). The corresponding 95\% confidence level upper limits $\left(\tau(\theta\right.$ Mus $)<0.68 \times 10^{-6}, \tau(\beta$ Sct $)<$ $\left.1.03 \times 10^{-6}\right)$ are also shown in Fig. 9 .

It is difficult to compare our results with the published OGLE analysis (Udalski et al. 2000), as our fields are located outside the region studied by this team. Moreover, the OGLE analysis efficiency and their observed optical depth are not yet available.

\section{Galaxy model, optical depth and event timescales}

From the position of the observer, the spatial distribution of the source stars and the density distribution of the deflectors, one can estimate the average optical depth toward a population of sources. We assume throughout this paper that the distance to the Galactic centre is $R_{\odot}=8.5 \mathrm{kpc}$. The velocity distributions and the deflector mass function are needed to estimate the event rate and obtain the Einstein radius crossing time distribution. 

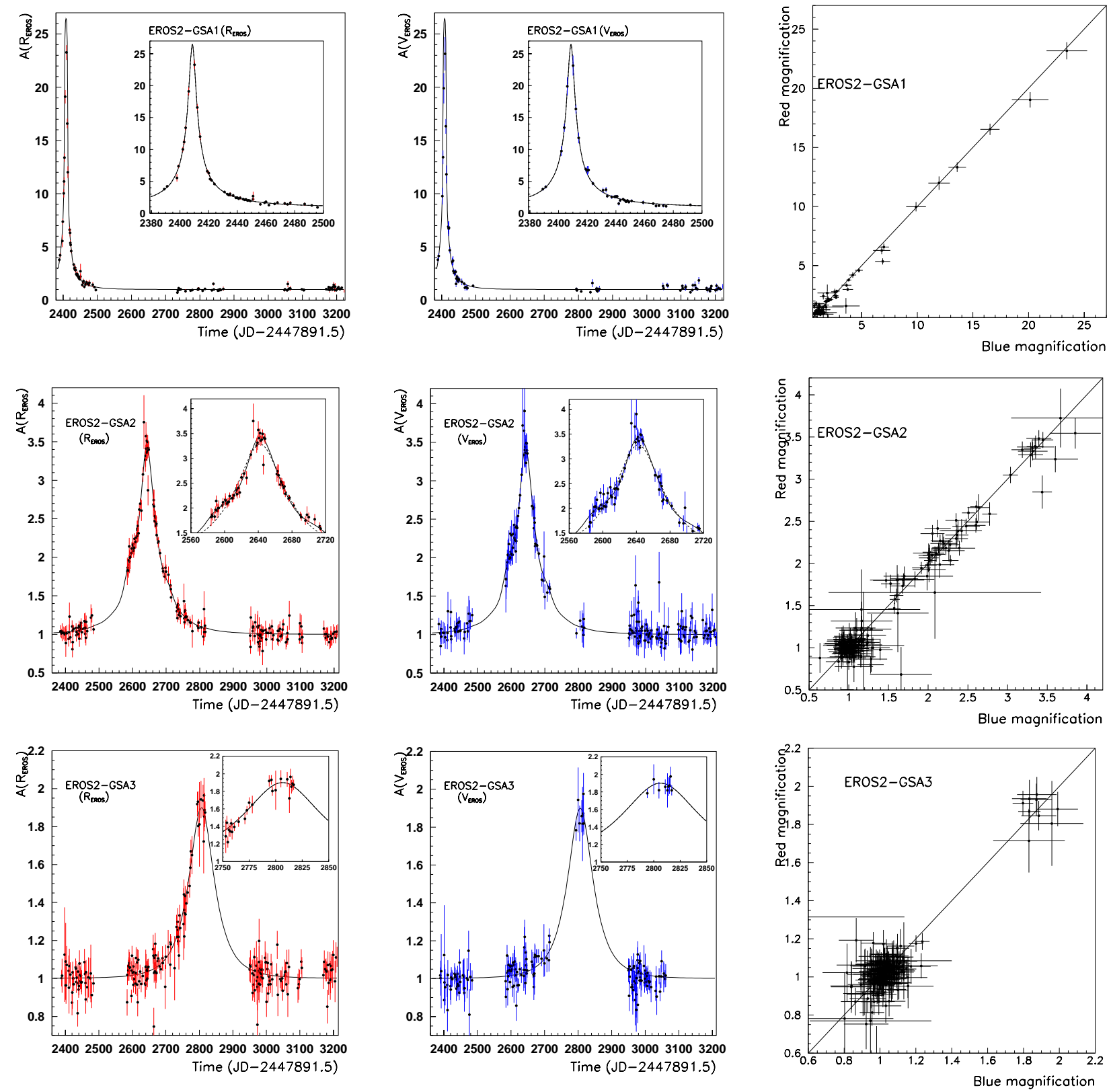

Fig. 7. Magnification curves of GSA1 to GSA3 microlensing candidates (left and middle panels). The fitted standard microlensing curve is superimposed (solid line). The insets show the light curve around maximum amplification time. On the right panel, red magnification is plotted versus the blue one for all pairs of simultaneous measurements. The solid lines indicate the magnification ratio equal to one (no chromatic effect).

\subsection{Spatial distribution of the sources}

In contrast with the Magellanic Clouds, the distance distribution of the monitored stars is poorly known, and should a priori vary with the limiting magnitude. In our detection conditions, the populations of stars used to obtain the optical depths measured in Sect. 5 are those described by the colour-magnitude diagrams of Fig. 6 . An analysis of these diagrams shows that their content is dominated by a population of source stars located $\sim 7 \mathrm{kpc}$ away, undergoing an interstellar extinction of about 3 magnitudes in $V_{\mathrm{J}}$ band (see Mansoux 1997 for more details). This distance estimate is in rough agreement with the distance to the spiral arms deduced from
Georgelin et al. (1994) and Russeil et al. (1998). We thus adopt $7 \mathrm{kpc}$ for the average distance of the source stars for the discussion presented in this paper.

\subsection{Deflectors}

\subsubsection{Density distribution}

We compute the expected optical depth using three component models for the distribution of the deflectors. A bulge, described by a barlike triaxial distribution, a disc, and a standard isotropic and isothermal halo. Two different models are considered. The first model (model 1) has a "thin" disc alone with a standard isotropic and isothermal halo. The second model (model 2) has a "thin" plus 

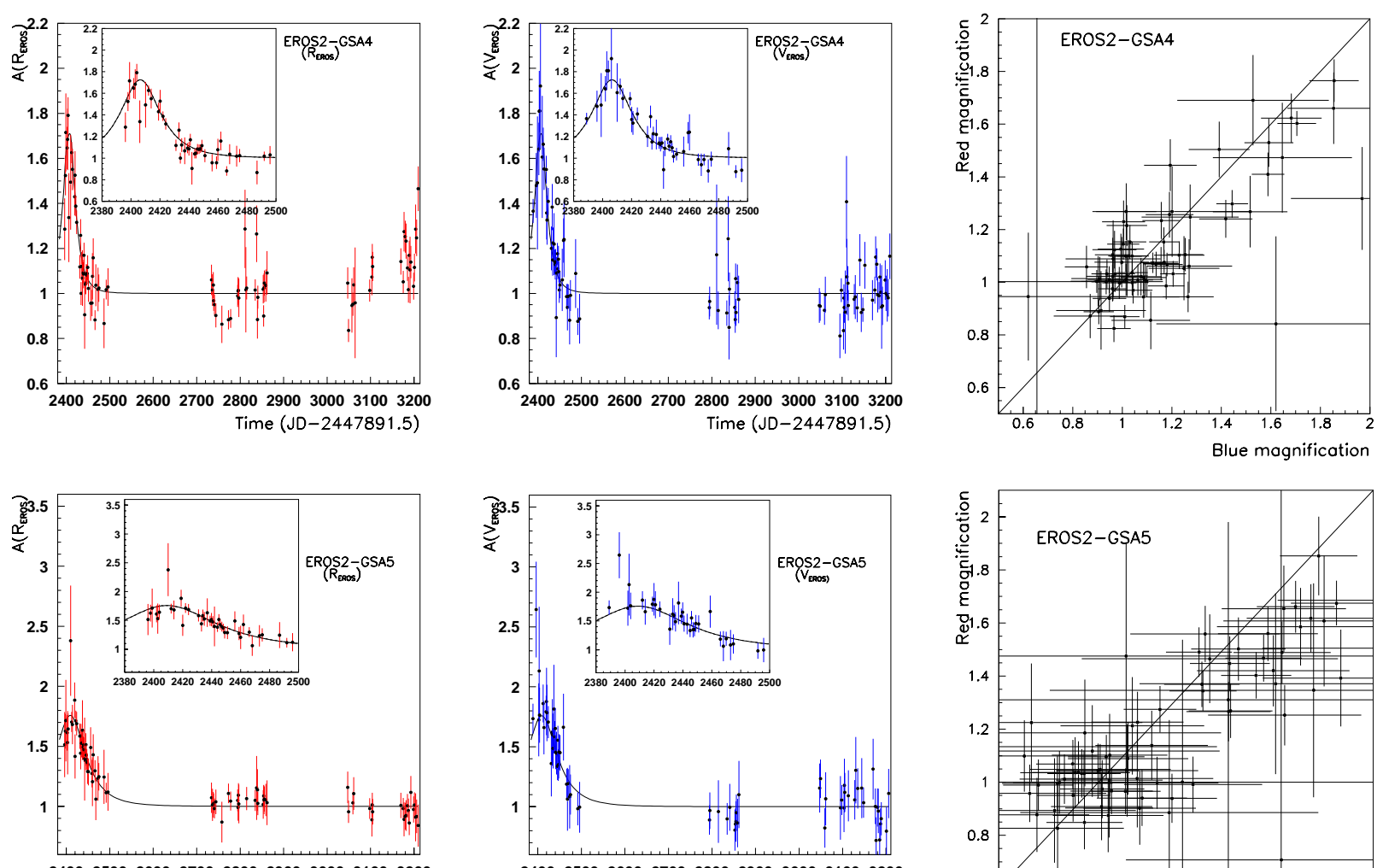

240025002600270028002900300031003200
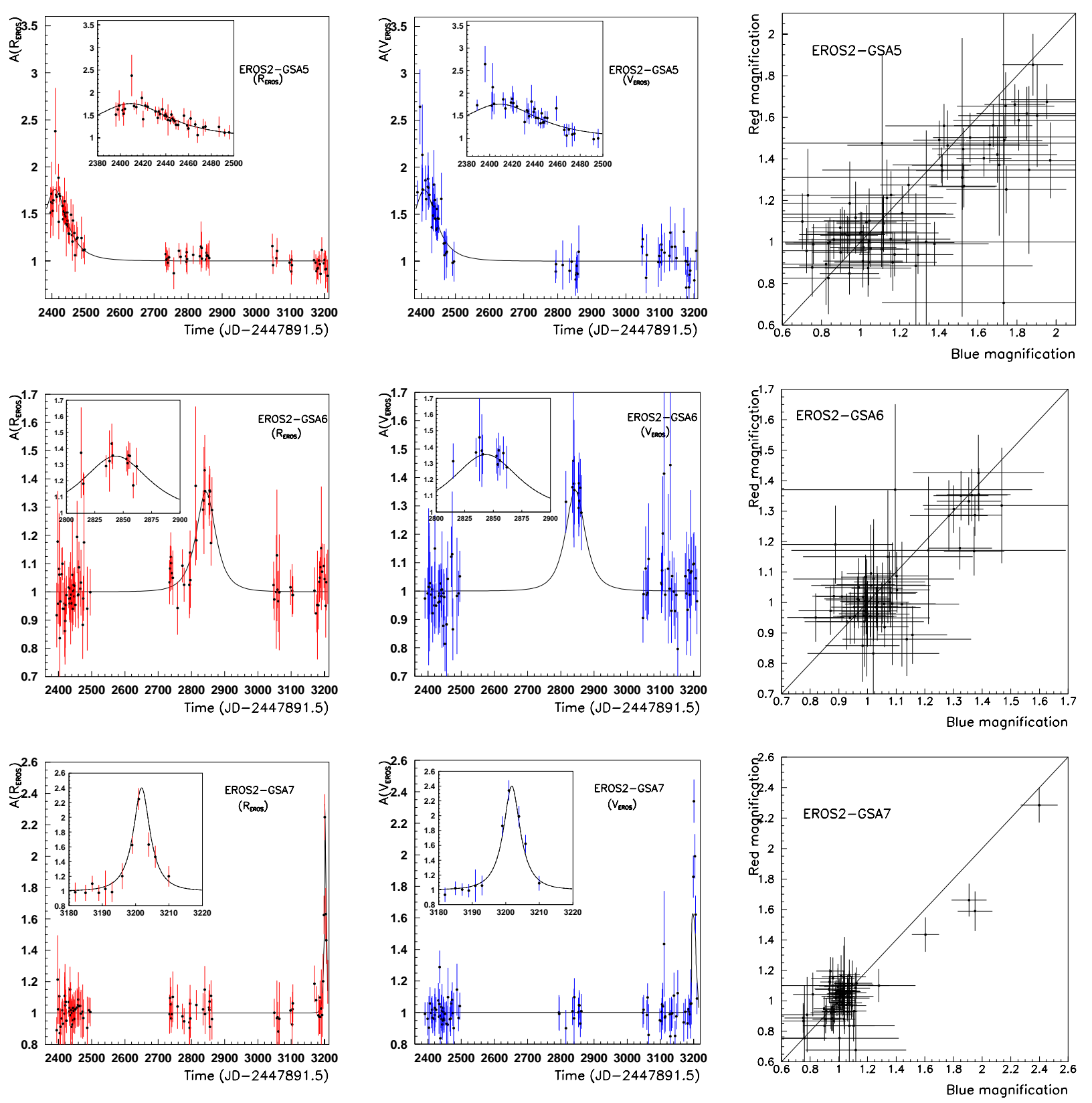

Fig. 8. Magnification curves of GSA4 to GSA7 microlensing candidates (left and middle panels). The fitted standard microlensing curve is superimposed (solid line). The insets show the light curve around maximum amplification time. On the right panel, red magnification is plotted versus the blue one for all pairs of simultaneous measurements. The solid lines indicate the magnification ratio equal to one (no chromatic effect). 
Table 4. Parameters of the galactic models used in this article and predictions for the rotation curve of the Milky Way.

\begin{tabular}{|c|c|c|c|}
\hline & Parameter & model 1 & model 2 \\
\hline Thin disc & $\begin{array}{l}\Sigma\left(M_{\odot} \mathrm{pc}^{-2}\right) \\
H(\mathrm{kpc}) \\
R(\mathrm{kpc}) \\
M_{\text {thin }}\left(\times 10^{10} M_{\odot}\right) \\
\end{array}$ & \multicolumn{2}{|c|}{$\begin{array}{c}50 \\
0.325 \\
3.5 \\
4.3\end{array}$} \\
\hline Thick disc & $\begin{array}{l}\Sigma\left(M_{\odot} \mathrm{pc}^{-2}\right) \\
H(\mathrm{kpc}) \\
R(\mathrm{kpc}) \\
M_{\text {thick }}\left(\times 10^{10} M_{\odot}\right)\end{array}$ & $\begin{array}{l}- \\
- \\
- \\
-\end{array}$ & $\begin{array}{l}35 \\
1.0 \\
3.5 \\
3.1\end{array}$ \\
\hline Bulge & $\begin{array}{l}a(\mathrm{kpc}) \\
b(\mathrm{kpc}) \\
c(\mathrm{kpc}) \\
M_{\mathrm{B}}\left(\times 10^{10} M_{\odot}\right)\end{array}$ & \multicolumn{2}{|c|}{$\begin{array}{c}1.49 \\
0.58 \\
0.40 \\
1.7 \\
\end{array}$} \\
\hline Halo & $\begin{array}{l}\rho_{\mathrm{h} \odot}\left(M_{\odot} \mathrm{pc}^{-3}\right) \\
R_{\mathrm{c}}(\mathrm{kpc}) \\
M\left(10^{10} M_{\odot}\right) \\
\text { within } 60 \mathrm{kpc} \\
\end{array}$ & $\begin{array}{c}0.008 \\
5.0 \\
51\end{array}$ & $\begin{array}{c}0.003 \\
5.0 \\
7\end{array}$ \\
\hline Predictions & $\begin{array}{l}\rho_{\odot}\left(M_{\odot} \mathrm{pc}^{-3}\right) \\
V_{\text {rot }} \text { at } \operatorname{sun}\left(\mathrm{km} \mathrm{s}^{-1}\right) \\
V_{\text {rot }} \text { at } 20 \mathrm{kpc} \\
V_{\text {rot }} \text { at } 60 \mathrm{kpc}\end{array}$ & $\begin{array}{c}0.085 \\
211 \\
203 \\
200\end{array}$ & $\begin{array}{c}0.098 \\
222 \\
180 \\
140\end{array}$ \\
\hline
\end{tabular}

a "thick" disc with a very light halo. The matter distribution in a disc is modeled in cylindrical coordinates by a double exponential:

$\rho_{D}(r, z)=\frac{\Sigma}{2 H} \exp \left(\frac{-\left(r-R_{\odot}\right)}{R}\right) \exp \left(\frac{-|z|}{H}\right)$,

where $\Sigma$ is the column density of the disc at the solar position, $H$ the height scale and $R$ the length scale of the disc.

The density distribution for the bulge - a barlike triaxial model - is taken from Dwek et al. (1995) model G2, given in Cartesian coordinates by:

$\rho_{\mathrm{B}}=\frac{M_{\mathrm{B}}}{6.57 \pi a b c} \mathrm{e}^{-r^{2} / 2}, r^{4}=\left[\left(\frac{x}{a}\right)^{2}+\left(\frac{y}{b}\right)^{2}\right]^{2}+\frac{z^{4}}{c^{4}}$,

where $M_{\mathrm{B}}$ is the bulge mass, and $a, b, c$ are the scale length factors. The bar major axis is tilted by $\phi=15^{\circ}$ with respect to the Sun-Galactic centre line. We use a standard isotropic and isothermal halo with a density distribution given in spherical coordinates by:

$\rho_{\mathrm{H}}(r)=\rho_{\mathrm{h} \odot} \frac{R_{\odot}^{2}+R_{\mathrm{c}}^{2}}{r^{2}+R_{\mathrm{c}}^{2}}$,

where $\rho_{\mathrm{h} \odot}$ is the local halo density, and $R_{\mathrm{c}}$ is the halo "core radius". The model parameters we use here are summarized in Table 4. As the precise morphology of the Galactic spiral arms is not known (see Englmaier \& Gerhard 1998), no spiral arm feature is included in our models.

\subsubsection{Mass function}

The mass function of the disc lenses is taken as the local stellar mass function given by Gould et al. (1997), which is derived from HST observations. For simplicity, we have used identical mass functions for the bulge and the disk lenses, although differences have recently been observed (see e.g. Zoccali et al. 2000). However, we have checked that changing the bulge mass function has a small impact on the expected event duration distribution. The mass function in the halo is still unknown and we assume that lenses belonging to the halo all have the same mass $M=$ $0.5 M_{\odot}$.

\subsection{Kinematic distributions}

\section{Observer}

For the solar motion with respect to the disc we have adopted the Standard Solar Motion (Delhaye 1965) which corresponds to a solar velocity of:

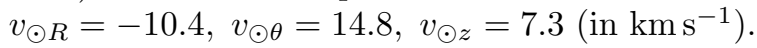

\section{Disc objects}

Disc objects are swept along by the similar global rotation as the Local Standard of Rest and the disc sources. We adopt the rotation curve of Brand \& Blitz (1993):

$V_{\text {rot }}(r)=V_{\text {rot }, \odot} \times\left[1.00762\left(\frac{r}{R_{\odot}}\right)^{0.0394}+0.00712\right]$,

with $V_{\text {rot, } \odot}=220 \mathrm{~km} \mathrm{~s}^{-1}$.

If all the microlensing actors belong to the disc, the average transverse velocity of the deflector relative to the line of sight is not large compared to the velocity dispersions. The first consequence is that we expect disc-lens/discsource events to have long timescales. The second consequence is that we have to take into account the peculiar velocity distributions to get the correct microlensing duration distributions. For the lenses we adopt the observed distribution of the local stars, described by an anisotropic Gaussian with the following velocity dispersions:

- for the thin disc: $\sigma_{r}=34 \mathrm{~km} \mathrm{~s}^{-1}, \sigma_{\theta}=28 \mathrm{~km} \mathrm{~s}^{-1}$ and $\sigma_{z}=20 \mathrm{~km} \mathrm{~s}^{-1}$

- for the thick disc: $\sigma_{r}=51 \mathrm{~km} \mathrm{~s}^{-1}, \sigma_{\theta}=38 \mathrm{~km} \mathrm{~s}^{-1}$ and $\sigma_{z}=35 \mathrm{~km} \mathrm{~s}^{-1}$.

As far as the source stars of the disc are concerned, they are assumed to be young objects with small peculiar velocities which we neglect.

\section{Bulge objects}

For sources or lenses located in the bulge we assume that the velocity distribution is Maxwellian. Then, the transverse velocity distribution is given by:

$f_{\mathrm{T}}\left(v_{\mathrm{T}}\right)=\frac{1}{\sigma_{\text {bulge }}^{2}} v_{\mathrm{T}} \exp \left(-\frac{v_{\mathrm{T}}^{2}}{2 \sigma_{\text {bulge }}^{2}}\right)$ 
with a velocity dispersion $\sigma_{\text {bulge }} \sim 110 \mathrm{~km} \mathrm{~s}^{-1}$.

\section{Halo objects}

For halo objects we consider also a Maxwellian velocity distribution with a typical velocity dispersion $\sigma_{\text {halo }} \sim$ $150 \mathrm{~km} \mathrm{~s}^{-1}$.

\subsection{Expected optical depth}

Figure 9 shows the expected optical depth up to $7 \mathrm{kpc}$ as a function of Galactic longitude for model 1 at the average Galactic latitude of our fields $\left(b=-2.5^{\circ}\right)$. As the main contribution comes from the thin disc (about 90\%), variations of the optical depth from field to field due to the range of 2 to $3^{\circ}$ in latitude can reach $\simeq 30 \%$ in the case of $\gamma$ Nor, and $\simeq 20 \%$ for the other targets. Note that at this Galactic latitude, the bulge and disc contributions are already reduced by a factor $\simeq 2$ with respect to zero latitude; moreover around zero longitude, the bulge contribution also depends dramatically on the distance to the target.

Assuming a standard halo made completely of compact objects would lead to a halo contribution of less than $10 \%$ to the Galactic spiral arms optical depth. Moreover the EROS measurements toward the LMC (Ansari et al. 1996; Alcock et al. 1998; Lasserre et al. 2000) and the SMC (Afonso et al. 1999) indicate that no more than $30 \%$ of this halo can be made of MACHOs lighter than $0.5 M_{\odot}$. Thus we will neglect the halo contribution in the following discussion.

The expected optical depths (without the halo contribution) for each monitored direction are reported in Table 5. The expected optical depth, averaged over the four directions, is $0.56 \times 10^{-6}$ for model 1 and $0.66 \times 10^{-6}$ for model 2. Our estimates of the disk optical depth are compatible with previous calculations by Grenacher et al. (1999).

Table 5. Expected and observed optical depth $\tau\left(\times 10^{6}\right)$, number of events $(N)$, average timescale $\left(\overline{t_{\mathrm{E}}}\right)$ and associated rms (in days) for each monitored direction, assuming that the distance to the source stars is $7 \mathrm{kpc}$. Note that in contrast to Fig. 9 the contribution of the dark halo has been removed.

\begin{tabular}{|cc|cccc|}
\hline \multicolumn{2}{|c|}{ Direction } & $\theta$ Mus & $\gamma$ Nor & $\gamma$ Sct & $\beta$ Sct \\
\hline \multirow{3}{*}{ model 1} & $\tau$ & 0.34 & 0.49 & 0.65 & 0.56 \\
& $N$ & 1.1 & 3.6 & 1.6 & 1.4 \\
& $t_{\mathrm{E}}$ & 72 & 67 & 53 & 66 \\
& $\sigma_{t_{\mathrm{E}}}$ & 48 & 45 & 42 & 47 \\
\hline \multirow{3}{*}{ model 2} & $\tau$ & 0.45 & 0.63 & 0.82 & 0.74 \\
& $N$ & 1.5 & 4.8 & 2.0 & 1.8 \\
& $-t_{\mathrm{E}}$ & 75 & 69 & 57 & 68 \\
& $\sigma_{t_{\mathrm{E}}}$ & 48 & 45 & 42 & 47 \\
\hline \multirow{6}{*}{ Observed } & $\tau$ & $<0.68$ & $0.27_{-0.17}^{+0.30}$ & $1.64_{-0.74}^{+0.92}$ & $<1.03$ \\
& $N$ & 0 & 2 & 5 & 0 \\
& $\bar{t}_{\mathrm{E}}$ & - & 84 & 40 & - \\
\hline
\end{tabular}

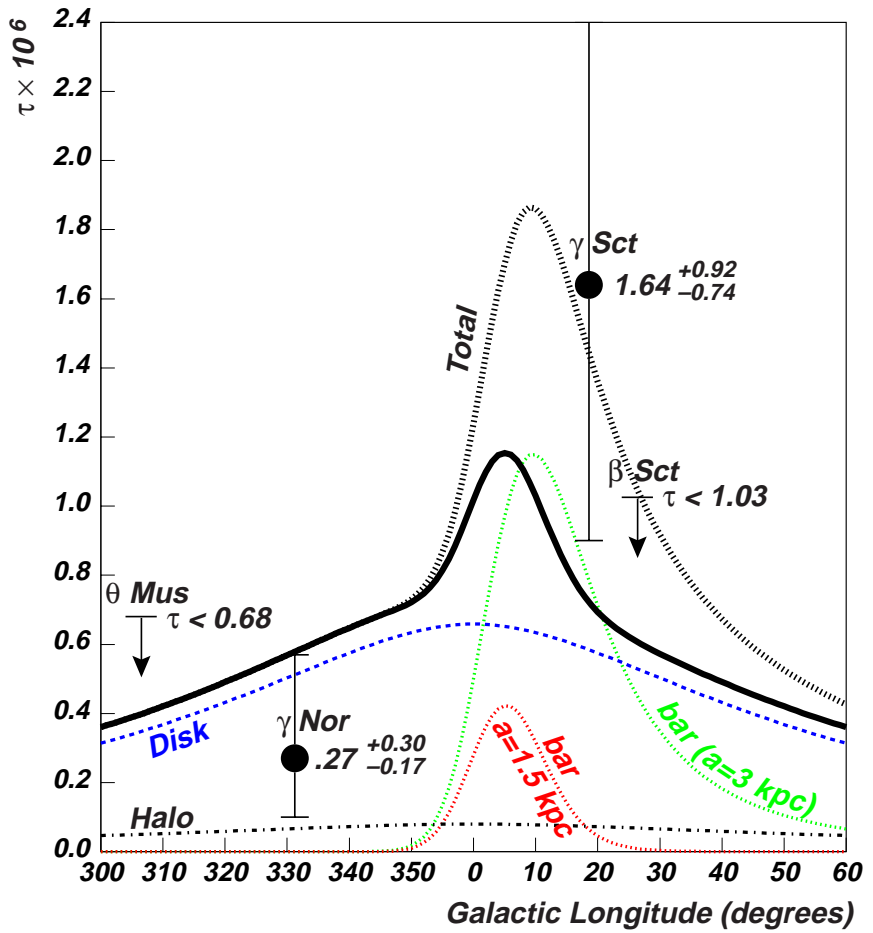

Fig. 9. Expected optical depth $\left(\times 10^{6}\right)$ up to $7 \mathrm{kpc}$ for the different components of the Milky Way as a function of the Galactic longitude at $b=-2.5$ for two values of the bar length parameter $(a)$, for model 1 . The thick lines indicate the total expected optical depth for the two values of bar length. The curve labeled Halo indicates the contribution of a halo made completely of MACHO's. The measured optical depths (or limits at $90 \%$ CL) are quoted for our 4 targets.

Table 6 shows the range of variation of the expected optical depth $\tau$, associated with the range of model parameters, toward $\gamma$ Sct (the only direction where the bulge contribution is significant). The domain of parameters we choose is very conservative and includes a wide variety of published estimates (Méra et al. 1998; Flynn \& Fuchs 1994; Sackett 1997 for the disc parameters; Stanek et al. 1994; Binney 2000). Note that the systematic uncertainty in $\tau$ due to the uncertainty in the distance to the sources a priori affects each direction in distinct ways. On the other hand, the systematic uncertainty due to the disc parameters affects the four monitored directions similarly.

Table 6. Variation of the optical depth with the change of parameters in the direction of $\gamma$ Sct (model 1).

\begin{tabular}{|c|c|cc|}
\hline & Parameters & Value range & $\Delta \tau / \tau$ in \% \\
\hline Dist. source & $D_{\mathrm{s}}(\mathrm{kpc})$ & {$[6 ; 8]$} & {$[-30 ;+40]$} \\
\hline \multirow{3}{*}{ Thin disc } & $\Sigma\left(M_{\odot} / \mathrm{pc}^{2}\right)$ & {$[25 ; 75]$} & {$[-45 ;+45]$} \\
& $H(\mathrm{kpc})$ & {$[0.25 ; 0.5]$} & {$[+10 ;-20]$} \\
& $R(\mathrm{kpc})$ & {$[2.0 ; 4.0]$} & {$[+100 ;-10]$} \\
\hline \multirow{3}{*}{ Bulge } & $M_{\mathrm{B}}\left(10^{10} M_{\odot}\right)$ & {$[0.7 ; 2.7]$} & {$[-7 ;+3]$} \\
& $a(\mathrm{kpc})$ & {$[0.7 ; 3.0]$} & {$[-10 ;+110]$} \\
& $\Phi\left(^{\circ}\right)$ & {$[0 ; 40]$} & {$[-10 ;+7]$} \\
\hline
\end{tabular}



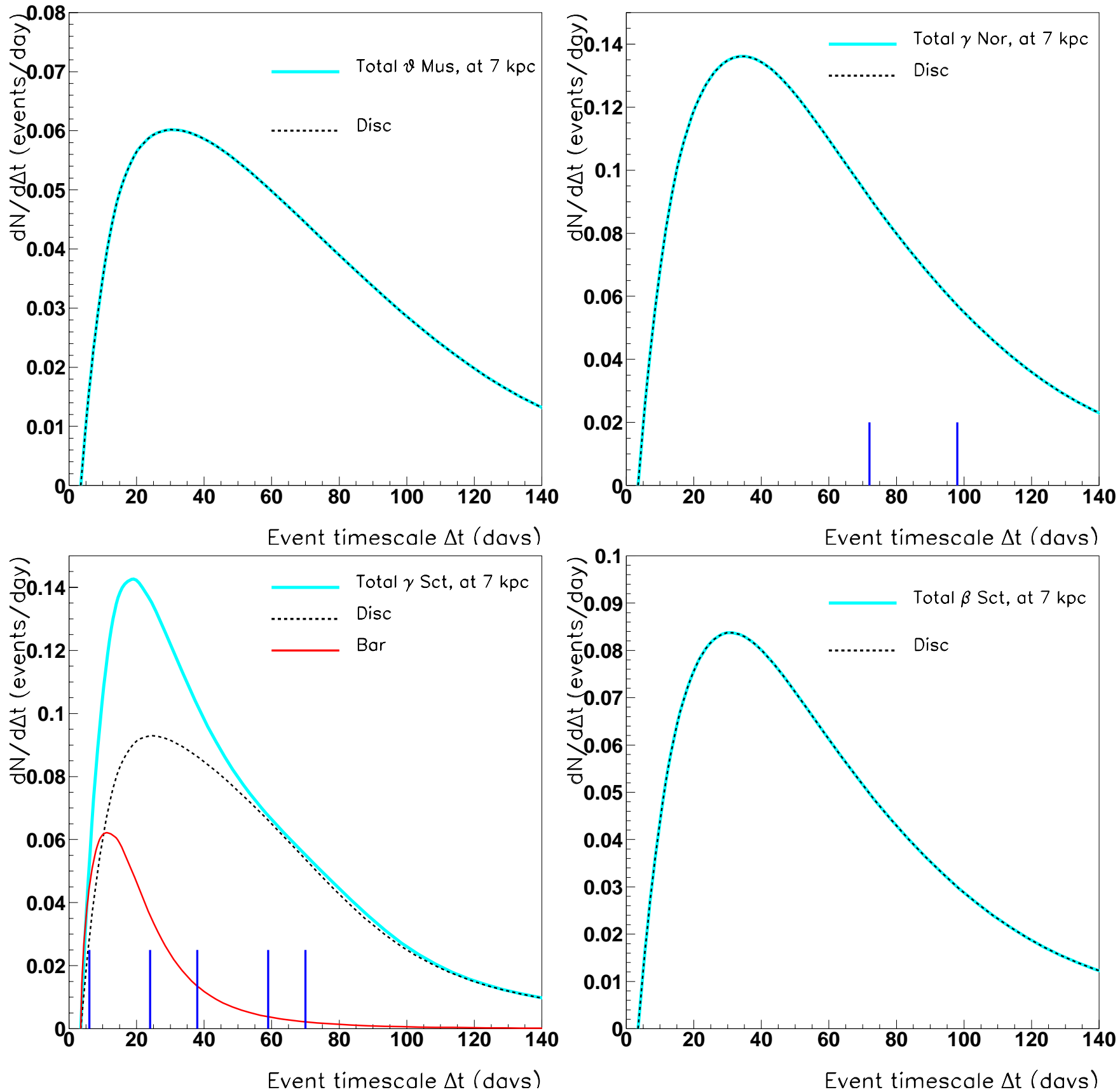

Fig. 10. $t_{\mathrm{E}}$ distribution of the events expected during the search period $T_{\text {search }}$ per $10^{7}$ monitored stars toward the four directions, when neglecting the halo contribution for model 1. These distributions take into account the detection efficiency $\epsilon\left(t_{\mathrm{E}}\right)$. The durations of the seven candidates are marked. Notice that the bulge contribution is negligible except toward $\gamma$ Sct.

As far as the bulge is concerned, the most sensitive parameter for the optical depth estimate toward $\gamma$ Sct is the semi-major axis length $a$ (see also Fig. 9). The three other targets are insensitive to the bar parameters.

\subsection{Event timescales and event rate}

Figure 10 shows the expected event duration distributions toward the four monitored directions. The durations of the seven selected candidates are also indicated. The predicted distributions are obtained using the kinematic characteristics and mass functions given above. As disc lenses have a low velocity relative to the line of sight, disc-disc events have longer timescales ( $\sim 60-70$ days) than bulgedisc events $(\sim 20$ days $)$.

In Table 5 we report the number of expected events and their mean duration $\left(\overline{t_{\mathrm{E}}}\right)$ for both models. The mean event duration expected toward $\gamma$ Sct is smaller due to a larger contribution from the bulge.

\section{Discussion}

The two targets $\gamma$ Sct and $\gamma$ Nor are located at nearly symmetric longitudes with respect to the Galactic centre. 
Therefore - assuming that the source distance is the same for both - they would experience the same systematic shift if the discs are different than modelled. Yet, we find an optical depth toward $\gamma$ Sct $\left(\simeq 1.64 \times 10^{-6}\right)$ significantly larger $($ at $\sim 2 \sigma)$ than toward $\gamma$ Nor $\left(\simeq 0.27 \times 10^{-6}\right)$. In addition, the average measured event timescale toward $\gamma$ Sct is 40 days, half of that observed for $\gamma$ Nor (84 days). A Student's Test of the two subsamples of events shows that the probability to get this difference (or worse) between the two averages is less than $9 \%^{2}$.

The optical depth asymmetry and the timescale difference we observe are then marginally compatible with what is expected from the bar contribution in our models. Apart from the statistical fluctuations, we discuss below the possible asymmetric systematic effects that could lead to differences between the event rates and characteristics toward the $\gamma$ Sct and the $\gamma$ Nor directions:

- The optical depth is very sensitive to the poorly known distance distribution of the monitored source stars, which depends on the star number density and the extinction along the line of sight. For example, changing the $\gamma$ Sct source star distances from $7 \mathrm{kpc}$ to $9 \mathrm{kpc}$ (resp $11 \mathrm{kpc}$ ) increases the expected optical depth from $0.75 \times 10^{-6}$ to $1.3 \times 10^{-6}$ (resp. $\left.1.93 \times 10^{-6}\right)$. However, this hypothesis cannot account for the shorter event durations observed toward $\gamma$ Sct;

- One cannot exclude the possibility of overdensities along the $\gamma$ Sct line of sight or underdensities toward $\gamma$ Nor, due to structures in the spiral arms. But here again, if such density fluctuations could explain differences of the optical depths, different kinematical behaviour should also be invoked to explain the difference in the $t_{\mathrm{E}}$ distributions;

- An increase in the bar length parameter (a) enhances the asymmetric contribution to the optical depth; Changing this parameter from $a=1.5$ to $a=3 \mathrm{kpc}$ changes the optical depth toward $\gamma$ Sct from 0.65 to $1.40 \times 10^{-6}$. In addition, bar induced microlensing events have a shorter time scale (as shown in Fig. 10), which would then explain the observed asymmetry in the event duration distribution.

\section{Conclusion}

We have searched for microlensing events with a duration ranging from a few days to a few months in four Galactic disc zones lying between $18^{\circ}$ to $55^{\circ}$ from the Galactic centre. A critical issue of such an investigation concerns the distances of the source stars - a question that could be addressed by performing a multiband wide-field photometric study of the target fields.

Our search has led to the discovery of seven events that can be interpreted as microlensing events due to

${ }_{2}$ This probability is very close to the probability to pick 2 unbiased events amongst the 7 , with an average duration larger than 84 days. massive compact objects. The estimated average optical depth inferred from this result is compatible with expectations from simple Galactic models. However, these events show different rates and time scales at different Galactic longitudes. These variations might point to a comparatively long Galactic bar.

Acknowledgements. We are grateful to D. Lacroix and the technical staff at the Observatoire de Haute Provence and to A. Baranne for their help in refurbishing the MARLY telescope and remounting it in La Silla. We are also grateful for the support given to our project by the technical staff at ESO, La Silla. We thank J. F. Lecointe for assistance with the online computing. We wish to thank also C. Nitschelm for his contribution to the data taking.

\section{References}

Afonso, C., Alard, C., Albert, J.-N., et al. (EROS Coll.) 1999, A\&A, 344, L63

Alcock, C., Akerlof, C. W., Allsman, R. A., et al. (MACHO Coll.) 1993, Nature, 365, 621

Alcock, C., Allsman, R. A., Axelrod, T. S., et al. (MACHO Coll.) 1995, ApJ, 445, 133

Alcock, C., Allsman, R. A., Alves, D., et al. (MACHO Coll.) 1997, ApJ, 479, 119

Alcock, C., Allsman, R. A., Alves, D., et al. 2000, ApJ, 541, 734

Alcock, C., Allsman, R. A., Alves, D., et al. (EROS Coll., MACHO Coll.) 1998, ApJ, 499, L9

Ansari, R., Cavalier, F., Moniez, M., et al. (EROS Coll.) 1996, A\&A, 314, 94

Ansari, R. 1996, Vistas Astron., 40(4), 1996

Aubourg, É., Bareyre, P., Bréhin, S., et al. (EROS Coll.) 1993, Nature, 365, 623

Binney, J. 2000, Proceedings of the Microlensing 2000, ASP Conf. Ser. [astro-ph/0004362]

Brand, J., \& Blitz, L. 1993, A\&A, 275, 67

Derue, F., Afonso, C., Alard, C., et al. (EROS Coll.) 1999, A\&A, 351, 87

Delhaye, J. 1965, in Galactic Structure (The University of Chicago Press)

de Vaucouleurs, G. 1964, in The Galaxy and the Magellanic Clouds, IAU Symp. 20, ed. F. J. Kerr, \& A. W. Rodgers (Camberra: Austr. Acad. Sci), 195

Dwek, E., Arendt, R. G., Hauser, M. G., et al. 1995, ApJ, 445, 716

Englmaier, P., \& Gerhard, O. 1999, MNRAS, 304, 512

Feldman, G. J., \& Cousins, R. D. 1998, Phys. Rev. D, 57, 3873

Flynn, C., \& Fuchs, B. 1994, MNRAS, 270, 471

Georgelin, Y. M., Amram, P., Georgelin, Y. P., et al. 1994, A\&AS, 108, 513

Gould, A., Bahcall, J. N., \& Flynn, C. 1997, ApJ, 482, 913

Grenacher, L., Jetzer, P., Strässle, M., \& De Paolis, F., 1999, A\&A, 351, 775

Holtzman, J. A., et al. 1993, AJ, 106, 1826

Han, C., \& Gould, A. 1995, ApJ, 449, 521

Harris, J., \& Zaritsky, D. 1999, AJ, 117, 2831

Hammersley, P. L., Garzon, F., Mahoney, T., LopezCorredoira, M., \& Torres, M. A. P. 2000, MNRAS, 317 L45 
Lasserre, T., Afonso, C., Albert, J. N., et al. (EROS Coll.) 2000, A\&A, 355, L39

Paczyński, B. 1986, ApJ, 304, 1

Mansoux, B. 1997, Ph.D. Thesis, Université Paris 7, LALCNRS/IN2P3 Report 97-19

Méra, D., Chabrier, G., \& Schaeffer, R. 1998, A\&A, 330, 953

Popowski, P., Alcock, C., Allsman, R. A., et al. 2000, To appear in Microlensing 2000, ASP Conf. Proc. [astro-ph/0005466]

Regnault, N. 2000, Ph.D. Thesis, Université Paris 7 LALCNRS/IN2P3 Report 00-65

Richer, H. B., \& Fahlman, G. G. 1992, Nature, 358, 383

Russeil, D., Amram, P., Georgelin, Y. P., et al. 1998, A\&AS, 130, 119

Sackett, P. 1997, ApJ, 483, 103
Stanek, K. Z., Mateo, M., Udalski, A., et al. (OGLE Coll.) 1994, ApJL, 429, 73

Udalski, A., Szymański, M., Kaluzny, J., et al. (OGLE Coll.) 1993, Act. Astr., 43, 289

Udalski, A., Szymański, M., Stanek, K. Z., et al. (OGLE Coll.) 1994, Act. Astr., 44, 165

Udalski, A., Szymański, M., Kubiak, M., et al. (OGLE Coll.) 1998, Act. Astr., 48, 1

Udalski, A., Zebrun, K., Szymański, et al. 2000, Acta. Astr., 50,1

Weiner, B. J., \& Sellwood J. A. 1999, [astro-ph/9904130]

Zhao, H., Spergel, D. N., \& Rich, R. 1996, MNRAS, 282, 175

Zoccali, M., Cassisi, S., Frogel, J. A., et al. 2000, ApJ, 530, 418 